\title{
A prospective randomized controlled study on the Suppression of Prostate Cancer by Naftopidil (SNAP)
} \author{
Homma $^{12}$ \\ ${ }^{1}$ Department of Urology, The University of Tokyo, Japan \\ ${ }^{2}$ Department of Urology, Teikyo University,School of Medicine, Japan \\ ${ }^{3}$ Clinical Research Support Center, The University of Tokyo Hospital, Japan \\ ${ }^{4}$ Department of Pathology, The University of Tokyo Hospital, Japan \\ ${ }^{5}$ Department of Urology, Mitsui Memorial Hospital, Japan \\ ${ }^{6}$ Department of Urology, Tokyo Teishin Hospital, Japan \\ ${ }^{7}$ Department of Urology, The Fraternity Memorial Hospital, Japan \\ ${ }^{8}$ Department of Urology, Graduate School of Medicine, Teikyo University, Japan \\ ${ }^{9}$ Department of Urology, National Center for Global Health and Medicine, Japan \\ ${ }^{10}$ Department of Urology, Graduate School of Medicine, The University of Tokyo, Japan \\ ${ }^{11}$ Department of Continence Medicine,The University of Tokyo, Japan \\ ${ }^{12}$ Japanese Red Cross Medical Center, Japan
}

Daisuke Yamada $^{1 *}$, Haruki Kume ${ }^{1}$, Hideyo Miyazaki ${ }^{2}$, Mototsugu Tanaka ${ }^{3}$, Munenori Takata $^{3}$, Yukari Uemura $^{3}$, Teppei Morikawa ${ }^{4}$, Yutaka Enomoto $^{5}$, Motofumi Suzuki ${ }^{6}$, Hiroaki Nishimatsu ${ }^{7}$, Tohru Nakagawa ${ }^{8}$, Tetsuya Fujimura ${ }^{9}$, Hiroshi Fukuhara ${ }^{10}$, Yasuhiko Igawa ${ }^{11}$ and Yukio

\begin{abstract}
Background: Naftopidil is an alpha-1 adrenergic receptor-blocking agent commonly used for benign prostatic hyperplasia (BPH) in Japan. Our previous retrospective study demonstrated a significantly lower incidence of prostate cancer in BPH men treated with naftopidil (1.8\%) compared with those with tamsulosin (3.1\%). Currently we are attempting to confirm the suppressive effect of naftopidil on prostate carcinogenesis in a prospective randomized study.

Methods: This multicenter, randomized, open-label clinical trial will enroll men who are proven negative for cancer after the first prostate biopsy at the University of Tokyo Hospital and its 11 affiliated hospitals. Upon agreement, these subjects will be randomly assigned to the oral naftopidil administration group (550 cases) or the non-administration control group (550 cases) and have serum prostate specific antigen (PSA) levels measured once a year for 5 years at longest. Prostate biopsy is to be repeated when the follow-up serum PSA level exceeds the precedent value. The primary endpoint is the time period to the diagnosis of prostate cancer. Prostate cancer-free survival calculated by Kaplan-Meier method is used to analyze inter-group differences by log-rank test.
\end{abstract}

Discussion: This is the first prospective randomized clinical study to verify whether naftopidil reduces the incidence of prostate cancer in men negative for cancer by first biopsy.

\section{Trial registration}

This study is registered as UMIN-CTR (study ID: UMIN000022862, date: 01/Dec/2016).

\begin{abstract}
Abbreviations: PSA: Prostate Specific Antigen, SNAP Suppression of Prostate Cancer by Naftopidil, TGF: Transforming Growth Factor
\end{abstract}

\section{Background}

The number of prostate cancer patients has been increasing rapidly worldwide. According to the statistics of the National Cancer Center (Tokyo, Japan), newly diagnosed prostate cancer patients in Japan were estimated as 98,400 in 2016, and is the leading incidence of male cancer [1]. The prevalence is expected to increase in the aging society, as prostate cancer is seen mostly in elderly males. Obviously, the most efficient method to reduce the prevalence of cancer is its prevention. However, supplementation of green tea or vitamin $\mathrm{E}$ have not been effective in reducing prostate cancer incidence in humans [2]. Five alpha reductase inhibitor showed a $25 \%$ reduction in incidence, although those patients who did develop prostate cancer had a higher grade tumor [3].

Naftopidil is an alpha-1 adrenergic receptor-blocking agent with greater affinity to alpha-1 adrenergic receptor subtypes A and D over B. It is commonly used in Japan for symptomatic benign prostatic hyperplasia, and its safety profile has been repeatedly confirmed during

Correspondence to: Daisuke Yamada, Department of Urology, The University of Tokyo, Japan, E-mail: dyamada-tky@umin.net

Key words: adrenergic, alpha, apoptosis, cancer, incidence, naftopidil, prevention, prostate, receptor, suppression

Received: February 13, 2018; Accepted: February 28, 2018; Published: March 05,2018 
its 17-year clinical use [4]. Interestingly, naftopidil exerts antitumor effects on prostate cancer cells in vitro [5]. It is known that some alpha-1 adrenergic receptor-blocking agents such as doxazosin or terazosin cause the growth suppression of cancer cells [6]. An anti-tumor effect was implicated with apoptotic activity rather than blocking alpha-1 adrenergic receptor signaling pathways [7]; tamsulosin, an alpha-1 adrenergic receptor-blocking agent without apoptotic activity, has no anti-cancer effects [8]. Clinically, a retrospective study by Harris et al. indicated the reduced incidence of prostate cancer in men administered doxazosin or terazosin compared with those not administered doxazosin or terazosin (1.6\% vs. $2.4 \%$ ) [9]. We have also demonstrated the inhibitory effect of naftopidil on prostate cancer development in an eight-year observational study; the prevalence in 766 men on naftopidil was $1.8 \%$, while that in 1,015 men on tamsulosin was $3.1 \%(\mathrm{p}<0.035)$ [10]. Furthermore, we confirmed that naftopidil induced apoptosis in prostatic cancer cell lines and human prostatic cancer tissues via inhibition of Smad2 phosphorylation-medicated transforming growth factor (TGF) beta activation [10]. Other inhibitory mechanisms proposed include activation of death receptors [11] and blocking tubulin formation [12]. The anti-proliferative effects of naftopidil on cell growth have been reported for human renal cancer cells, bladder cancer, cervical cancer, and malignant pleural mesothelioma [10,13-16].

These potential anti-tumor effects and the good safety profile of naftopidil prompted us to conduct this prospective randomized clinical trial to investigate the preventive effects of naftopidil on prostate cancer development.

\section{Methods/Design}

\section{Outline of the study}

This study is a randomized, open-label, controlled, parallel, clinical trial and has been approved by the research ethics committee of the Graduate School of Medicine and Faculty of Medicine, The University of
Tokyo (P2016007) and independent institutional review boards of each affiliation. The study contents are explained by descriptive documents and written consent, and patients from whom consent is obtained then is registered and randomized (Figure 1). Naftopidil is administered to patients assigned to the oral naftopidil administration group and not to those assigned to the non-administration control group, and patients have their serum prostate specific antigen (PSA) levels measured once a year for 5 years at the longest. Prostate biopsy is to be repeated when the follow-up serum PSA level exceeds the value obtained before the previous biopsy. Cases where the serum PSA level does not increase and prostatic needle biopsy is not performed are regarded as negative. For prostatic needle biopsy, standardized methods in each facility are used (however, biopsy should be performed on eight areas or more) so that the same biopsy is performed for both groups. Development of prostate cancer is assumed as a main evaluation item, and differences in the incidence of prostate cancer in both groups will be examined.

\section{Subject assignment method}

Assignment of subjects to each administration group is performed by the central registration method using the Viedoc TM system (PCG Solutions, Uppsala, Sweden) with stratified block randomization, with a 1:1 ratio of subjects in the oral naftopidil administration group and non-administration control groups. The assignment factors are age $(<70$ years, $\geq 70$ years), serum PSA level $(<7, \geq 7)$, facilities (each cooperative facility) and volume of prostate $(<30 \mathrm{~g}, \geq 30 \mathrm{~g})$. The assignment list is created and stored by the University of Tokyo Hospital, The Clinical Research Support Center, and The Central Management Unit and is closed to the investigators and sub investigators.

\section{Subject patient}

\section{(1) Inclusion criteria}

1) Patients whose cancer is not proved by the first prostatic needle biopsy (eight spots or more) performed within the past three months.

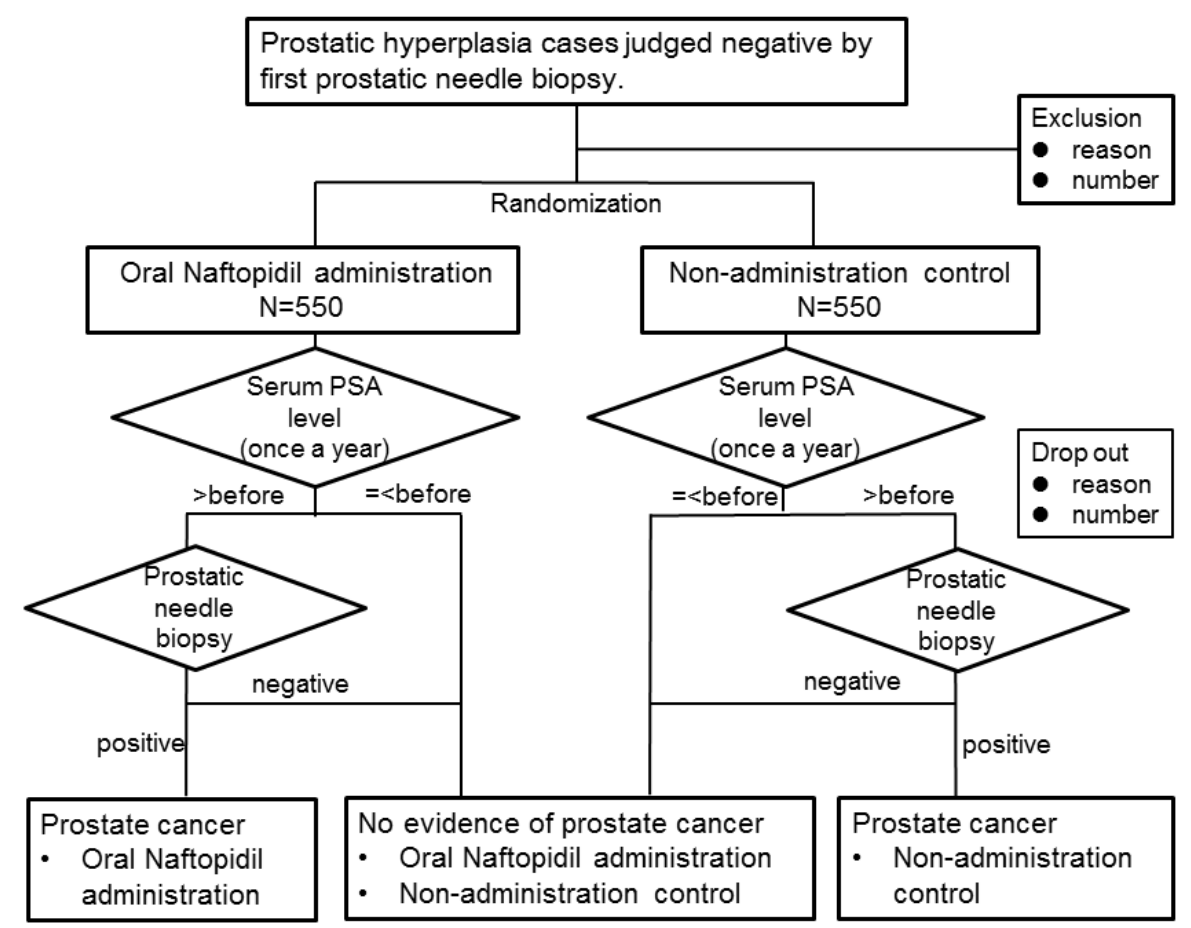

Figure 1. Outline of the prospective randomized controlled study on Suppression of Prostate Cancer by Naftopidil (SNAP) 
2) Male patients aged $\geq 50$ years and $<80$ years at the time when consent is acquired.

3) Patients whose most recent serum PSA levels are abnormal before prostatic needle biopsy (> $3.0 \mathrm{ng} / \mathrm{ml}$ for $50-64$ year olds, $>3.5$ $\mathrm{ng} / \mathrm{ml}$ for $65-69$ year olds, and $>4.0 \mathrm{ng} / \mathrm{ml}$ for $\geq 70$ year olds).

4) Patients who are given sufficient description about participation in this study and who understand it, providing documentary consent by their own free will.

5) Patients with prostatic hyperplasia.

\section{(2) Exclusion criteria}

1) Patients who were given a diagnosis of prostate cancer or atypical small acinar proliferation. Prostatic intraepithelial neoplasia is accepted.

2) Patients with a history of oral administration of naftopidil, antiandrogenic medicine, or 5 alpha-reductase inhibitors.

3) Patients with severe dysuria accompanied with prostatic hyperplasia (states such as urinary retention, urethral catheter placement, and intermittent urethral catheterization).

4) Patients who have a prostate surgical history.

5) Patients who are currently taking steroid medicine on consecutive days.

6) Patients with acute prostatitis.

7) Patients with active urogenital infection.

8) Patients who have a urethral catheter.

9) Patients with severe hepatic dysfunction (aspartate transaminase or alanine transaminase $>100 \mathrm{IU} / \mathrm{L}$ ). $\mathrm{mg} / \mathrm{dl})$.

10) Patient with severe renal dysfunction (serum creatinine $>2.0$

11) Patients with a history of malignant tumor except prostate cancer (however, cases that were cured radically and with a non-disease period of $>$ two years will be accepted).

12) Patients who are judged inappropriate by investigators.

\section{Planned study participation period}

Registration period is 2 years and administration period is to be 3 to 5 years. However, in cases where prostate cancer is detected, the study is to end at that point.

\section{Dosage and administration of the study drug and administration period}

The study drug is to be administered orally with an appropriate amount of water. The administration is to continue until the end of the study. The dose is $25 \mathrm{mg}$ a day in the early period (more than two weeks) of administration, and then increased to $50 \mathrm{mg}$ a day in the later phase. However, the dose can be changed within the range of $25-75 \mathrm{mg}$ depending on the symptoms and the number of administrations per day is not limited.

\section{Provisions for combined medicine (therapy)}

\section{(A) Prohibited concomitant medications (therapy)}

The following medicines (therapy) that may influence the development of prostate cancer and serum PSA levels are prohibited.
However, when required clinically, priority will be given to its use and this study is to end.

1) Daily use of steroid medicine (however, external medicines are accepted. Furthermore, if the period of its use is within one month in total, temporary use is acceptable).

2) Antiandrogenic drugs (Chlormadinone, Allylestrenol, Gestonorone).

3) Five alpha-reductase inhibitors (Dutasteride, Finasteride).

4) Alpha-1 adrenergic receptor-blocking agents (Silodosin, Urapidil, Terazosin, Doxazosin, Bunazosin, Phentolamine, and Prazosin) (however, if the period of its use is within one month in total, temporary use is acceptable).

5) Anti-neoplastic drugs

6) Prostate operation (transurethral resection of the prostate, holmium laser enucleation of the prostate, photo-selective vaporization of the prostate by laser, transurethral microwave thermotherapy, or high-intensity focused ultrasound).

7) Urethral catheter placement, intermittent urethral catheterization (however, if the period of its use is within one month in total, temporary use is acceptable).

\section{(B) Authorized drugs}

The following agents are thought not to influence the development of prostate cancer and their continuous concomitant use is allowed.

1) Alpha-1 adrenergic receptor-blocking agents (Tamsulosin).

2) Antihypertensive agents except for alpha-1 adrenergic receptorblocking agents.

\section{Endpoints}

\section{(A) Primary endpoint}

The primary evaluation item is the period from the day of registration to diagnosed as prostate cancer.

\section{(B) Secondary endpoints}

\section{1) Malignancy of prostate cancer}

Malignancy will be evaluated by the cancer property (Gleason score) and extent (positive number of needle biopsies / number of needle biopsies).

2) Rate of apoptotic cells in immunostained specimens of needle biopsy.

3) Doubling time of serum PSA levels from baseline.

4) Cancer incidence in other organs.

\section{(C) Safety evaluation items}

The incidence of adverse events and side effects will be compared.

\section{Pathological examinations}

Malignancy, expanse, and percentage of apoptosis of the prostate cancer are evaluated at the Department of Pathology at the University of Tokyo Hospital.

\section{Statistical analysis}

Based on a previous study [10], the prostate cancer incidence hazard ratio of naftopidil for tamsulosin was 0.46 and therefore, a relative risk 
reduction of $54 \%$ was estimated. To verify the superiority of the oral naftopidil administration group compared to the non-administration control group, when the hazard ratio, significant level (both sides) and power of the test are set to $0.6,5 \%$ and $80 \%$, respectively, 120 events (prostate cancer development) are necessary for both groups. To secure 120 events, 1,030 cases are needed in total for both groups with an event incidence of $6.9 \%$ in the oral naftopidil administration group with PSA $>4 \mathrm{ng} / \mathrm{ml}$ (subgroup analyses of previous report [10]). The number of cases where the dropout rate is $15 \%, 20 \%$, and $25 \%$ over five years are $1,098,1,124$, and 1,152 cases, respectively. Therefore, the target number of cases was determined to be 1,100 cases for both groups.

Incidence of prostate cancer in each group will be obtained by the Kaplan-Meier method, and intergroup comparison will be performed by log-rank test. Furthermore, hazard ratio of the oral naftopidil administration group and the non-administration control group and the $95 \%$ confidence intervals (CI) will be calculated by the Cox proportional-hazard model. Significant level is assumed as 5\% for both sides. Malignancy will be evaluated by properties and extent of the cancer and the intergroup comparison will be performed by $t$-test. The doubling time of PSA will be calculated from serum PSA values measured every year.

\section{Interim analysis}

Interim analysis for safety and efficacy will be performed two and a half years after registration or when the event expressions of 60 cases are confirmed, whichever comes first. In the case that the following are recognized in the interim analysis, the Independent Data Monitoring Committee can urge withdrawal of the study to the Executive Committee, so as not to let the unfavorable treatment group continue: (1) efficacy of the oral naftopidil administration group is significantly superior to that of the non-administration control group, and (2) efficacy of the oral naftopidil administration group is adversely inferior to that of the non-administration control group, and superiority cannot be verified by continuing the study.

\section{Quality control / quality assurance}

A data management and monitoring system are established for quality control. Audits for quality assurance are conducted. The audit members are not engaged in the study or monitoring.

\section{Discussion}

Prospective controlled clinical studies on cancer prevention require a large sample size and a long study period. Preventive measure with high efficacy and safety as well as selection of susceptible study population is of critical importance. Our study use naftopidil, an alpha-1 adrenergic receptor-blocking agent, as the preventive medication, and recruit cancer-negative men at the first prostate biopsy as the target sample.

Preventive effects of naftopidil on prostate cancer development are demonstrated by observational clinical studies. Non-human studies also indicate its anti-proliferative effect on multiple cancer cells in vitro or in vivo in mice $[10,16]$. It is fortunate that naftopidil distributes preferentially in the prostate; the intra-prostatic concentration after an oral dose of $20 \mathrm{mg} / \mathrm{kg}$ in rats reached 50-100 $\mu \mathrm{g} / \mathrm{g}$ (1-24 hours), which was highest among all organs studied [17]. These concentrations are equivalent to $128-256 \mu \mathrm{M}$, given the molecular weight of naftopidil (392) and a conversion rate of $1 \mathrm{~g}=1 \mathrm{ml}$. The clinical dosage (25$75 \mathrm{mg}$ ) corresponds to $0.5-1.5 \mathrm{mg} / \mathrm{kg}$ for a $50-\mathrm{kg}$ man. Presuming that naftopidil has a similar distribution in rats and humans, the concentration of naftopidil in the human prostate is expected be 6.4$12.8 \mu \mathrm{M}$, an effective concentration in vitro [18]. Indeed, a previous study [10] reported greater in vitro apoptosis in cancer cells at $10 \mu \mathrm{M}$ and a larger number of apoptotic cells in prostatic cancer tissues of patients taking $25-75 \mathrm{mg}$ naftopidil orally. Naftopidil is proven to be a safe drug. According to the medical package insert of naftopidil, adverse reactions were observed in 721 cases $(3.28 \%)$ from a total of 22,013: wobbling in 209 cases $(0.95 \%)$, dizziness at standing up in 93 cases (0.42\%), hypotension in 44 cases $(0.20 \%)$, and stomach discomfort in 43 cases $(0.20 \%)$.

The target population is set for men negative for prostate cancer at the first biopsy despite elevated serum PSA values. The participants are to be randomly divided into two groups, equivalent in terms of cancer susceptibility by adjusting multiple confounding factors. We will adjust possible confounders for the development of prostate cancer such as age ( $<70$ years, $\geq 70$ years), serum PSA level $(<7, \geq 7)$, facilities (each cooperative facility) and volume of prostate $(<30 \mathrm{~g}, \geq 30 \mathrm{~g})$ as the assignment factors. Prostate volume is a negative contributor for prostate cancer, partially because a cancer focus in a large prostate is likely to be overlooked. The probability of cancer detection at the second biopsy in men negative at the first biopsy is reported to be as high as $30 \%[19]$. This probability enables us to anticipate the event (cancer detection) at $15 \%$ for the non-administration control group. Presuming reduction by naftopidil is $7.5 \%$, the sample number of 1,100 for the study included 550 in the oral naftopidil administration group and 550 in the non-administration control group.

A concern of the study design is the non-blinded fashion for allocation. This may result in less vigorous indication of prostate biopsy by the investigators' and the consequent decreased cancer detection rate in the naftopidil group. Strict compliance to the protocol should be retained. However, patients in the control (non-administered) group may take naftopidil for preventive intention. A thorough explanation should be given when acquiring consent. Symptomatic progression in men of control group may incur ethical concerns, which is to be managed by tamsulosin.

This study has some limitations, even if we can demonstrate the inhibitory effects of naftopidil on prostate carcinogenesis. This study involves men who are negative at first prostatic needle biopsy, thus the results cannot be generalized. However, these men would be the most appropriate candidates for preventive measures thus our results will meet a medical need. Men in the control group are allowed to take tamsulosin, and its unknown effects on prostate cancer cannot be negated. The dosage of naftopidil follows the dose approved for benign prostatic hyperplasia. It will remain unknown whether the dosage is optimum.

This study is the first prospective study to verify whether naftopidil, an alpha-1 adrenergic receptor-blocking agent, prevents prostate cancer in a high-risk population of men.

\section{Declarations}

\section{Ethics approval and consent to participate}

All patients signed an informed consent for the intervention, data acquisition and data evaluation. This study is based on ICH-GCP guidelines. Moreover, the study is conducted in compliance with the "Ethical guideline of medical system study for humans" and the latest Helsinki Declaration. The study investigators are to have the execution plan, description document and consent form reviewed by the Ethics Committees of each facility and to acquire permission for 
the study from the directors of the hospitals in each facility (approved: Graduate School of Medicine and Faculty of Medicine, The University of Tokyo; P2016007, National Center for Global Health and Medicine; NCGM-G-002154-00, Mitsui Memorial Hospital; MEC2016-21, Tokyo Teishin Hospital; Rin-1018, Tokyo Metropolitan Geriatric Hospital and Institute of Gerontology; R16-44, Tokyo Metropolitan Police Hospital; 16-a20, Japanese Red Cross Medical Center; 745, Ome Municipal General Hospital; 38, Tokyo Metropolitan Bokutoh Hospital; 76, The Fraternity Memorial Hospital; 169, Saitama Medical Center;16-21, Kamagaya General Hospital; TGE00782-064). In compliance with the Helsinki Declaration and ethical guidelines for clinical studies, the investigators will make their best effort for subjects to receive the best prevention, diagnosis, and treatment offered even after the study is completed. The study has been registered in UMIN-CTR (Study ID: UMIN000022862, date 01/Dec/2016) and will be updated depending on changes in the study plan and progress of the study. The results of the study will be registered as soon as the study is completed.

\section{Availability of data and material}

The data that support the findings of this study are available from Tokyo University and Asahi Kasei Pharma Corporation but restrictions apply to the availability of these data, which were used under license for the current study, and so are not publicly available. Data are however available from the authors upon reasonable request and with the permission of Tokyo University and Asahi Kasei Pharma Corporation.

\section{Competing interests}

\section{(1) Competing interests for the entire study}

This clinical study is conducted with a research fund provided from Asahi Kasei Pharma Corporation based on the academia led type consignment study contract. Asahi Kasei Pharma Corporation offers information on the study drugs and is not involved with execution of the research, analysis, and report. Moreover, no funds were received from other enterprises related to the study drugs.

\section{(2) Competing interests for the clinical departments}

The clinical departments of each facility concerned receive research funds according to the registration of the study subjects for consignment study of The University of Tokyo and each facility, based on the academia-led type consignment study contract between The University of Tokyo and Asahi Kasei Pharma Corporation.

\section{(3) Competing interests for researchers}

Y. I. received donations from Asahi Kasei Pharma Corporation and Kyorin Pharmaceutical Co., Ltd., although these were not for conducting this study but for the promotion of general academic studies. H. F. received a research grant from Asahi Kasei Pharma Corporation although this was not for conducting this study but for other research.

\section{Funding}

This clinical study is conducted with research funds provided by the Asahi Kasei Pharma Corporation based on the academia led type consignment study contract.

\section{Authors' contributions}

DY is the principal investigator of the study. YH is responsible for conducting the study overall. DY drafted the first version of the protocol, which was subsequently further developed by HK, HM, MT, MT, YE, MS, HN, TN, TF, HF, and YH. YU is responsible for the statistical analyses. TM will oversee the pathological analyses. All authors reviewed the manuscript and approved the final version.

\section{Acknowledgements}

We would like to acknowledge Atsushi Kato, Akira Ishikawa, Yasushi Kondo, Yasushi Nagase, Takeshi Azuma, Yutaka Kasuya, Hisashi Matsushima, Yukio Yamada, Taro Murata, Kenji Yoda, Keishi Kashibuchi, Takashi Murata, Takamitsu Tachikawa and Toshikazu Okaneya, who interacted with the ethics committee at each facility and who will recruit patients and collect follow-up data, Jinpei Miyakawa who will be assist pathological analyses and Maki Kobayashi and Mari Hirano who conduct office work.

\section{References}

1. http://ganjoho.jp/reg_stat/statistics/stat/short_pred.html. Acessed on Nov 23, 2016.

2. Özten-Kandaş N, Bosland MC (2011) Chemoprevention of prostate cancer: Natural compounds, antiandrogens, and antioxidants - In vivo evidence. J Carcinog 10: 27. [Crossref]

3. Theoret MR, Ning YM, Zhang JJ, Justice R, Keegan P, et al. (2011) The risks and benefits of $5 \alpha$-reductase inhibitors for prostate-cancer prevention. $N$ engl J Med 365: 97-99. [Crossref]

4. Gotoh M, Kamihira O, Kinukawa T, Ono Y, Ohshima S, et al. (2005) Urological Clinical Trial G: Comparison of tamsulosin and naftopidil for efficacy and safety in the treatment of benign prostatic hyperplasia: a randomized controlled trial. BJU Int 96: 581-586. [Crossref]

5. Kanda H, Ishii K, Ogura Y, Imamura T, Kanai M, et al. (2008) Naftopidil, a selective alpha-1 adrenoceptor antagonist, inhibits growth of human prostate cancer cells by G1 cell cycle arrest. Int J Cancer 122: 444-451. [Crossref]

6. Rinner I, Kukulansky T, Skreiner E, Globerson A, Kasai M, et al. (1994) Adrenergic and cholinergic regulation of apoptosis and differentiation of thymic lymphocytes. $A d v$ Exp Med Biol 355: 113-117.

7. Benning CM, Kyprianou N (2002) Quinazoline-derived alpha1-adrenoceptor antagonists induce prostate cancer cell apoptosis via an alpha1-adrenoceptorindependent action. Cancer Res 62: 597-602. [Crossref]

8. Kyprianou N, Benning CM (2000) Suppression of human prostate cancer cell growth by alpha1-adrenoceptor antagonists doxazosin and terazosin via induction of apoptosis. Cancer Res 60: 4550-4555. [Crossref]

9. Harris AM, Warner BW, Wilson JM, Becker A, Rowland RG, et al. (2007) Effect of alpha1-adrenoceptor antagonist exposure on prostate cancer incidence: an observational cohort study. J Urol 178: 2176-2180. [Crossref]

10. Yamada D, Nishimatsu H, Kumano S, Hirano Y, Suzuki M, et al. (2013) Reduction of prostate cancer incidence by naftopidil, an alpha1-adrenoceptor antagonist and transforming growth factor-beta signaling inhibitor. Int J Urol 20: 1220-1227.

11. Gotoh A, Nagaya H, Kanno T, Nishizaki T (2012) Antitumor action of alpha (1)-adrenoceptor blockers on human bladder, prostate and renal cancer cells. Pharmacology 90: 242-246.

12. Ishii K, Sugimura Y (2015) Identification of a new pharmacological activity of the phenylpiperazine derivative naftopidil: tubulin-binding drug. $J$ Chem Biol 8: 5-9. [Crossref]

13. Nakagawa YU, Nagaya H, Miyata T, Wada Y, Oyama T, et al. (2016) Piperazine-based Alpha-1 AR Blocker, Naftopidil, Selectively Suppresses Malignant Human Bladder Cells via Induction of Apoptosis. Anticancer Res 36: 1563-1570. [Crossref]

14. Mikami K, Nagaya H, Gotoh A, Kanno T, Tsuchiya A, et al. (2014) Naftopidil is usefu for the treatment of malignant pleural mesothelioma. Pharmacology 94: 163-169. [Crossref]

15. Masachika E, Kanno T, Nakano T, Gotoh A, Nishizaki T (2013) Naftopidil induces apoptosis in malignant mesothelioma cell lines independently of alpha1-adrenoceptor blocking. Anticancer Res 33: 887-894. [Crossref]

16. Iwamoto Y, Ishii K, Sasaki T, Kato M, Kanda H, et al. (2013) Oral naftopidil suppresses human renal-cell carcinoma by inducing $\mathrm{G}$ (1) cell-cycle arrest in tumor and vascular endothelial cells. Cancer Prev Res (Phila) 6: 1000-1006. [Crossref] 
Yamada D (2018) A prospective randomized controlled study on the Suppression of Prostate Cancer by Naftopidil (SNAP)

17. Liu X, Zhang X, Huang J, Rong Y, Luo C, et al. (2015) Enantiospecific determination of naftopidil by RRLC-MS/MS reveals stereoselective pharmacokinetics and tissue distributions in rats. J Pharm Biomed Anal 112: 147-154. [Crossref]

18. Chiou WL, Barve A (1998) Linear correlation of the fraction of oral dose absorbed of 64 drugs between humans and rats. Pharm Res 15: 1792-1795. [Crossref]
19. Nelson AW, Harvey RC, Parker RA, Kastner C, Doble A, et al. (2013) Repeat prostate biopsy strategies after initial negative biopsy: meta-regression comparing cancer detection of transperineal, transrectal saturation and MRI guided biopsy. PLoS One 8: e57480. [Crossref]

Copyright: (C2018 Yamada D. This is an open-access article distributed under the terms of the Creative Commons Attribution License, which permits unrestricted use, distribution, and reproduction in any medium, provided the original author and source are credited. 